\title{
Update on the Use of Arthroscopy in Kienböck's Disease Management
}

\section{Actualización de la utilidad de la Artroscopia en el Manejo de la Enfermedad de Kienböck}

René Jorquera Aguilera1,2,3 Camila Azócar Sanhueza 3,4,5

1 IWAS Faculty, IWC, Chile

2 Orthopedics and Traumatology Department, Universidad Andrés Bello, Chile

${ }^{3}$ Hand Team, Artroscopia y Microcirugía Clínica Indisa, Santiago de Chile, Chile

${ }^{4}$ Orthopedics and Traumatology Department, Universidad de Chile, Chile

${ }^{5}$ Hand Team, Hospital Clínico Mutual de Seguridad de Santiago de Chile, Chile

Rev Iberam Cir Mano 2020;48:119-127.
Address for correspondence Camila Azócar Sanhueza, MD, Santa María, 1810, Providencia, Santiago, Chile (e-mail: camiazocars@gmail.com).

\section{Abstract \\ Keywords \\ - Kienböck's disease \\ - lunate avascular necrosis \\ - wrist arthroscopy \\ - scaphocapitate arthrodesis \\ - partial wrist fusion.}

Kienböck's disease is a clinical-radiological condition that can permanently affect wrist functionality and the patient's quality of life. Proper, pertinent diagnosis and treatment are required to avoid salvage procedures. Arthroscopic techniques have emerged as an important tool for the treatment of certain stages of the disease.

We present a review of the literature and propose a treatment algorithm according to the stage of the disease, including arthroscopic techniques. We detail the surgical technique of arthroscopic scaphocapitate arthrodesis, and the functional outcomes obtained by our group.

La enfermedad de Kienböck es una condición clínico-radiológica que puede afectar de manera permanente la funcionalidad de la muñeca y la calidad de vida del paciente. Se requiere de un diagnóstico y manejo oportuno para evitar la necesidad de cirugías de salvataje. Las técnicas artroscópicas han aparecido como importante herramienta para el manejo en ciertas etapas de la enfermedad.

Realizamos una revisión de la literatura y proponemos un esquema de manejo según estadío de la enfermedad incluyendo técnicas artroscópicas. Detallamos la técnica quirúrgica de la artrodesis escafocapitate artroscópica y resultados funcionales obtenidos por nuestro grupo de trabajo.

- artrodesis escafocapitate

- artrodesis parcial del carpo.

received

October 5, 2020

accepted

October 15, 2020
DOI https://doi.org/

10.1055/s-0040-1721047. ISSN 1698-8396.
Copyright $\odot 2020$ Thieme Revinter

Publicações Ltda, Rio de Janeiro, Brazil
License terms

(ㄷ) (i) $\ominus$ (\$) 


\section{Introduction}

Kienböck's disease, described in 1910 by radiologist Robert Kienböck as a lunate chondromalacia, ${ }^{1-3}$ represents a real challenge for hand surgeons since the current literature remains unclear regarding its etiology, natural evolution, and specific treatment. ${ }^{1-8}$

This is a progressive clinical-radiological condition of the wrist involving the lunate bone, which can fragment and collapse due to avascular necrosis. ${ }^{2}$ It mostly affects young, working people, ${ }^{4}$ usually at the dominant extremity. Bilateral involvement is rare. ${ }^{9}$

Although Kienböck's disease etiology and pathogenesis remains unknown, ${ }^{1,2,4,5}$ several potential causes have been postulated, including repetitive trauma, vascular changes and lunate anatomical (extravascular) differences as the most studied entities. ${ }^{1,2,4,5}$

Patients often present hand complaints evolving for months ${ }^{10}$ which are usually confused with other conditions. Fifty per cent of these patients have a history of minor trauma. ${ }^{9}$ The clinical presentation is characterized by dorsal wrist pain, ${ }^{2-5}$ commonly progressive, and potentially associated to limited hand extension and loss of hand strength. ${ }^{5}$

A patient with these features requires a correct diagnosis to base a therapeutic proposal tailored to individual needs. Initial imaging study with simple radiographs (anteroposterior [AP] and profile [P] images) of the wrist may be sufficient to establish the diagnosis in patients with advanced Kienböck's disease, but it is of little use in its first stages, in which wrist synovitis predominates. In these cases, magnetic resonance imaging (MRI) is critical for diagnosis and differential diagnosis; in addition, MRI provides fundamental information for therapeutic decisions, especially in assessing lunate vascular conditions ${ }^{3}$ and adjoining structures, like joint cartilage.

Radiologically, characteristic images of the disease have been described both for the lunate and remaining carpal bones. In 1977, Lichtman et al. ${ }^{11}$ described a classification system that has been used in the literature for the last 50 years. This system is centered on simple wrist radiographies detailing the progressive stages of the disease, which are based mainly on lunate deformities and involvement of other carpal bones. In 2006, Bain and Begg ${ }^{12}$ proposed an arthroscopic classification for Kienböck's disease based on the status of the midcarpal and radiocarpal joints cartilage. In recent years, these authors have proposed a new classification method incorporating clinical features and previous systems in an updated treatment algorithm. ${ }^{3}$

The arthroscopic assessment allows for a better evaluation of the true status of the lunate and carpal bones joint cartilage regarding the pathophysiological progression of the intraosseous damage associated with cartilage injury. Arthroscopy can provide accurate information to base the proper initial or definitive surgical treatment. ${ }^{8}$ In addition, it has a fundamental role at the therapeutic decision-making process.

Based on Lichtman and Bain ${ }^{3,7}$ and considering mainly the lunate bone status and the secondary wrist joint involvement, a therapeutic scheme can be proposed. Early Kienböck's disease treatment usually aims a favorable environment for lunate revascularization; in advanced disease, however, treatment aims to diminish the pain caused by synovitis and to preserve movement.

This paper discusses the arthroscopic technique used for wrist joints diagnosis and assessment. We also suggest a therapeutic scheme according to the stage of the condition and describe the arthroscopic techniques most often used in patients with Kienböck's disease.

\section{Arthroscopic Evaluation of the Wrist with Kienböck's Disease}

We believe that a comprehensive initial arthroscopic evaluation of the wrist is required to accurately diagnose its joint status and to indicate the proper treatment for these patients. This is especially true in more advanced stages since the surgical technique depends on these findings. We frequently indicate surgery based on the clinical and imaging evaluation with no previous arthroscopy. In these interventional cases, and considering our preferences for surgical treatment, the procedure begins with a diagnostic arthroscopy for joint evaluation and the appropriate operative technique is contingent on the findings in each case. To do so, we often have a therapeutic scheme focused on lunate involvement and the general status of the wrist based on the Litchman classification. ${ }^{12}$ For the surgical act, we usually try to have all the supplies and instruments required for different techniques; although there are no substantial differences in such resources, the surgeon must have the training, experience and versatility in a wide range of proposed techniques after joint surfaces assessment, which is a critical point for making decisions about the selected approach.

Our assessment starts with a standardized diagnostic wrist arthroscopy technique for radiocarpal evaluation using the classic 1-2, 3-4 and 6R portals and then the midcarpal joint evaluation using the midcarpal ulnar (MCU) and midcarpal radial (MCR) portals.

The main concern at this stage is joint cartilage status assessment. Through the radiocarpal joint, the quality of the distal radial joint surface in its two pits and the proximal joint facets of the scaphoid and lunate bones are evaluated. In addition, the intrinsic scapholunate and lunotriquetral ligament structures, the extrinsic ligaments, and the triangular fibrocartilage complex are examined. It is important to record specifics about any lunate cartilage softening (mainly in its most proximal, radial region), wear with subchondral bone involvement, and lunate fragmentation or overt fracture, with coronal fractures with the worst prognosis. ${ }^{13}$ At the midcarpal joint, emphasis is placed on capitate and scaphoid evaluation for signs of osteoarthritis; the lunate must be assessed for the same phenomena described for the radiocarpal joint.

In both joints, it is also very important to evaluate the ligament status in a targeted manner, looking for any associated instability that could compromise surgical outcomes.

Following a complete evaluation of the midcarpal joint, the patient is classified according to the Bain and Begg system. ${ }^{11}$ This will allow us to indicate the proper surgical technique to be performed. 


\section{Therapeutic Decision-Making}

The therapeutic decision making in a patient with Kienböck's disease is complex and must consider a number of individual factors, including age, functionality, dominance, work and recreational activities, and concomitant conditions. Patients are usually young, athletic, workers with important functional requirements.

This is why, when surgery is indicated, we try to perform minimally invasive procedures that spare healthy structures and mobility as much as possible. The therapeutic goal is to prevent lunate and carpal bones collapse in the initial stages, to restore the architecture in advanced stages and to improve function and pain in terminal stages of the disease.

Therapeutic options include multiple surgical techniques specifically aimed at the Kienböck's disease stage at the time of the surgical decision, either with early minor lunate fragmentation and collapse or more advanced degrees of lunate devascularization and radial and midcarpal joints involvement.

Treatment is aimed at reducing pain, protecting the vulnerable lunate bone, and providing an ideal environment for revascularization. ${ }^{3}$ In more advanced stages, with significant lunate involvement and secondary joint involvement, the treatment is aimed at reducing pain and trying to preserve some degree of wrist mobility through techniques such as partial carpal arthrodesis or proximal carpectomy. ${ }^{3,7}$ In terminal stages, with advanced wrist involvement, definitive treatment consists of salvage techniques, such as total wrist arthrodesis or arthroplasty. ${ }^{3}$

On the other hand, it is important to recognize our own technical limitations and remember that these procedures are complex and require a learning curve to avoid complications and poor clinical outcomes due to surgical failure.

\section{Management in Early Stages}

Early stages refer to those with a structurally undamaged lunate and no cartilage changes at both the midcarpal and radiocarpal joints. These patients are classified as stages $0, \mathrm{I}$ and II according to Litchman ${ }^{12}$ and stage 0 according to Bain and Begg. ${ }^{11}$

The initial treatment for early stages of the disease consists of no less than 6 weeks of immobilization and symptomatic pain management followed by a period of rehabilitation. ${ }^{14}$ The goal is to stop lunate necrosis evolution and to detect revascularization signs at an MRI scan (- Figure $\mathbf{1}$ ).

Surgical treatment is indicated to patients with no symptomatic or imaging improvement even after a prolonged period of immobilization. In such cases, surgery aims to reduce the load and stress over the lunate bone, with different surgical techniques for mechanical or biological lunate decompression.

Surgical options for mechanical decompression include distal radius wedge osteotomy and capitate shortening osteotomy, both with good long-term clinical outcomes. ${ }^{15}$ We do not perform these routine techniques in these patients, and we prefer biological decompression procedures.
There are two techniques for lunate biological decompression: the forage-type lunate decompression and the metaphyseal decompression of the distal radius described by Illarramendi et al., ${ }^{16}$ who reported good long-term outcomes. ${ }^{17}$ We routinely perform this latter technique, either alone or associated with a vascularized graft in Lichtman stage II. Through a bone window in the lateral border of the distal radius, the metaphyseal cancellous bone is compressed with a spoon towards the distal radial walls. This would produce an increase in the revascularization potential of the lunate due to the local activation of reparative factors resulting in focused hyperemia and neovascularization stimulation. It is a simple, reproducible procedure that does not alter the normal anatomy of the wrist.

As previously mentioned, in patients with Lichtman stage II, we suggest the distal radial vascularized graft technique along with the decompression procedure. ${ }^{18}$ We use a dorsal graft under the 4-5 extensor compartment and protect it with a short arm cast and a scaphocapitate Kirschner wire for 6 weeks (-Figure 2 ). Our series of seven patients followed-up for 3 years yielded good outcomes, with 100\% revascularization, immediate pain reduction, mobility range improvement and activities resuming.

In summary, patients in stages 0 , I and II are conservatively treated for 2 to 3 months. In case of medical treatment failure, we suggest a metaphyseal decompression technique for patients in stages 0 and $I$ and its association with a vascularized graft for patients in stage II.

\section{Management in Intermediate Stages}

Intermediate disease consists in Lichtman stage IIIA, with complete lunate involvement but sparing the remaining carpal bones and with no wrist collapse.

In these patients, arthroscopy is essential to assess the lunate joint cartilage status and indicate the proper surgical treatment. In patients classified as Bain and Begg stage 1, we indicate a proximal carpectomy. This is an infrequent scenario and we usually find some distal radial joint damage which contraindicates this technique. For these patients, who correspond to Bain and Begg stage 2a, we propose a scaphocapitate arthrodesis as the technique of choice.

Scaphocapitate arthrodesis was initially described as an open technique ${ }^{7,19}$ for scaphoid nonunion management. It transfers the load to the radial column, decompressing the damaged central column. It can be performed when the lunocapitate joint facet is compromised, a stage in which proximal carpectomy is contraindicated and with few therapeutic options.

In recent years, arthroscopic partial carpal arthrodesis techniques have been developed ${ }^{2,3,5-8}$ with the advantages of being minimally invasive and less associated with soft tissue damage. ${ }^{8}$ Scaphocapitate arthrodesis may be performed with arthroscopic assistance and its associated advantages. The arthroscopic technique is described under "Surgical Technique for Scaphocapitate Arthrodesis with Arthroscopic Assistance". 


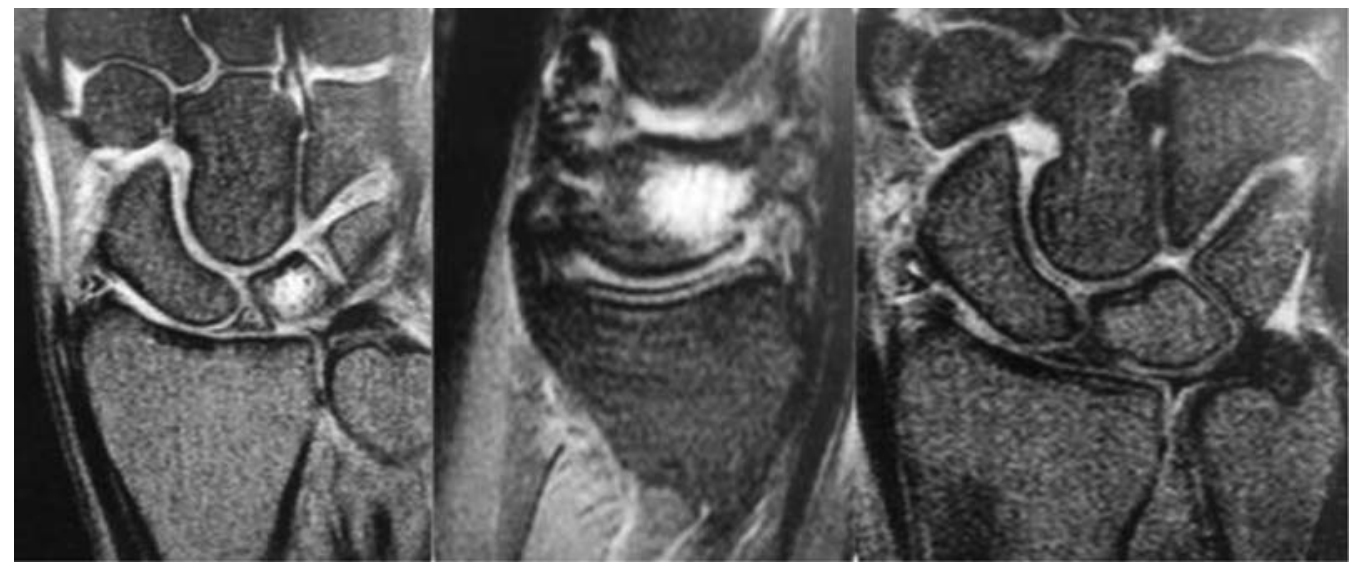

Figure 1 Clinical case of a 32-year-old female patient with dominant limb diagnosed with Kienböck's disease in stage 0 to I per Lichtman and 0 per Bain/Begg. She was treated with a short arm cast for 3 months. The previous and final magnetic resonance imaging scans are shown, revealing lunate vascular changes.

\section{Management in Advanced Stages}

Patients with Lichtman stages IIIA, IIIB and IIIC and joint involvement 2-4 per the Bain and Begg classification are considered advanced cases. In these patients, since both the radiocarpal and midcarpal joints are affected, therapeutic options are limited, and partial fusions are considered the treatment of choice.

We suggest the technique of scaphocapitate arthrodesis with arthroscopic assistance for these patients. We believe that this is an effective technique to reduce painful symptoms while allowing for some range of mobility of the wrist.
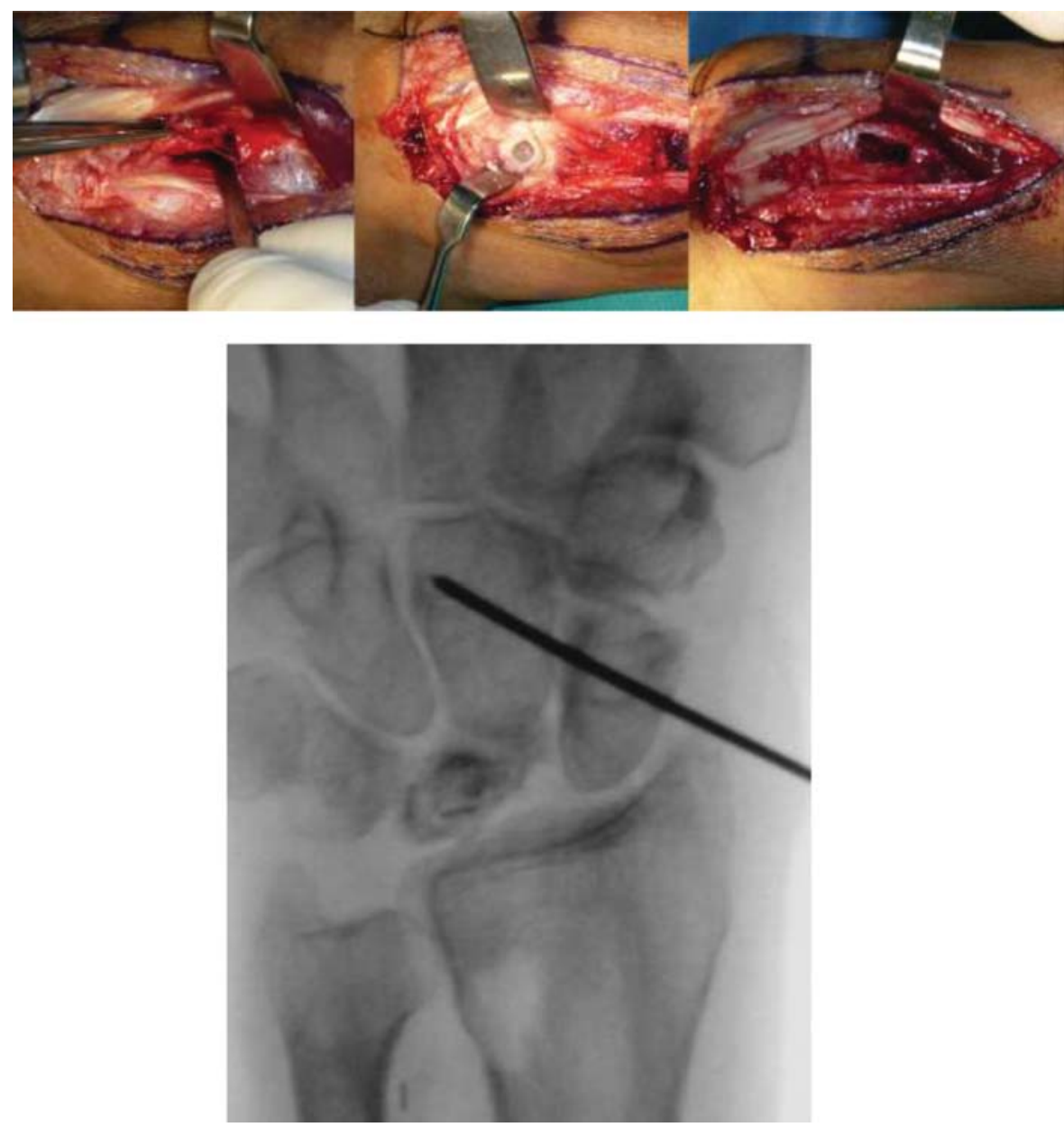

Figure 2 Surgical technique to obtain a vascularized graft from the 4-5 extensor compartment. The graft and its pedicle, the perforation in the recipient lunate and the surgical outcome are shown. The postoperative anteroposterior radiograph of the wrist shows the scaphocapitate guidewire protected with a Kirschner wire and a short arm cast for 6 weeks. 


\section{Surgical Technique for Scaphocapitate Arthrodesis with Arthroscopic Assistance}

Wrist positioning and traction are performed according to the standard arthroscopy technique. A 2.7-mm optics with $30^{\circ}$ angulation is required. Diagnostic arthroscopy must be wet and performed with no ischemia because surgical arthrodesis can be demanding and must not exceed 120 minutes. Epinephrine in a 1:1000 dilution is injected directly at the portals, with no intra-articular drug injection. After diagnostic arthroscopy, limb ischemia is performed using a proximal tourniquet at $100 \mathrm{~mm} \mathrm{Hg}$ above the patient's systolic pressure. At this phase, we suggest a mixed approach, using wet arthroscopy with free fluid rinse (connected to two bags of Ringer Lactate solution, $6 \mathrm{~L}$ each), and dry arthroscopy according to the surgical step.

- Diagnostic radiocarpal arthroscopy: A diagnostic arthroscopy is performed as previously described. In these patients, we expect to find a fragmented lunate with damaged joint cartilage ( - Figure $3 \mathbf{A}$ ). The scaphoid is expected to be intact, as well as the scaphoid facet of the distal radius. The scapholunate ligament, which is usually injured, must be visualized. It is important to visualize the radioscaphocapitate (RSC) ligament, which must be intact for this technique. We suggest a wet arthroscopy for this stage to better visualize all the structures requiring evaluation.

- Diagnostic midcarpal arthroscopy: Midcarpal portals are used to visualize the midcarpal joint cartilage. In this stage, the lunate is usually fragmented and there is evident damage to the joint cartilage ( - Figure $3 \mathbf{3 B}$ ). The proximal pole of the capitate presents obvious signs of chondromalacia (-Figure 3C) and a complete scapholunate lesion is often present. This step is performed under wet arthroscopy.

\section{- Arthroscopic fragmentation of the lunate and partial} lunectomy: Using a $2.9-\mathrm{mm}$ burr and a $2.9-\mathrm{mm}$ shaver, the lunate is fragmented and partially resected. This is done through both joints using the midcarpal and radiocarpal portals. The lunate is partially removed since this approach does not allow for a complete resection. With the shaver, the scapholunate and lunotriquetral ligaments are also resected, leaving a space in the place of the lunate. It is important to perform the lunectomy using a mixed arthroscopy, interspersing dry and wet phases for proper joint cleaning and to reduce the heat generated by the instruments.

- Scaphoid and capitate cartilage denudation: An accessory radial midcarpal portal (MCR-A) is made over the scaphoid and used as a "working portal". With the optics at the MCU, we work through the MCR and MCR-A portals and the scaphocapitate joint cartilage is completely denuded using the burr and shaver. At this stage, it is important to have an adequate flow of intra-articular solution for cleaning and dissipating the heat generated. Since there is a space in the first carpal row due to the previous excision of the lunate, it is possible to pass the optics through the radiocarpal portals for visualization and to use the midcarpal portals as working portals.

- Complete lunate resection through the 3-4 portal: Traction is released, and the limb is horizontally positioned on the table with the hand in pronation. The 3-4 portal is enlarged through a mini-open approach of sufficient size to pass a gouge-type forceps (-Figures $\mathbf{4}$ and $\mathbf{5}$ ). Through it, the previously fractionated lunate is completely resected, and, under fluoroscopy, we ensure complete bone excision and the absence of intra-articular free bone fragments.

- Bone graft extraction and Illarramendi technique: A 3$\mathrm{cm}$ approach is made on the lateral aspect of the distal radius by blunt dissection between the first and second extensor compartments. Bone can be accessed protecting structures at risk. A cortical window is carved into the distal radial metaphysis and a cancellous graft is extracted with a spoon. Next, a metaphyseal decompression of the distal radius is performed as described by Illarramendi et $\mathrm{al}^{12}$ (- Figure 6).

- Stabilization with guidewires: Under fluoroscopic view, two scaphocapitate guidewires were implanted in a parallel arrangement in the same position headless compression screws were subsequently fixed for future arthrodesis. Screw guidewires are used; we used a 3.0$\mathrm{mm}$ distal screw and a $2.4-\mathrm{mm}$ proximal screw. After placing both guidewires in the correct position, they are completely withdrawn from the capitate, leaving them only at the scaphoid thickness.

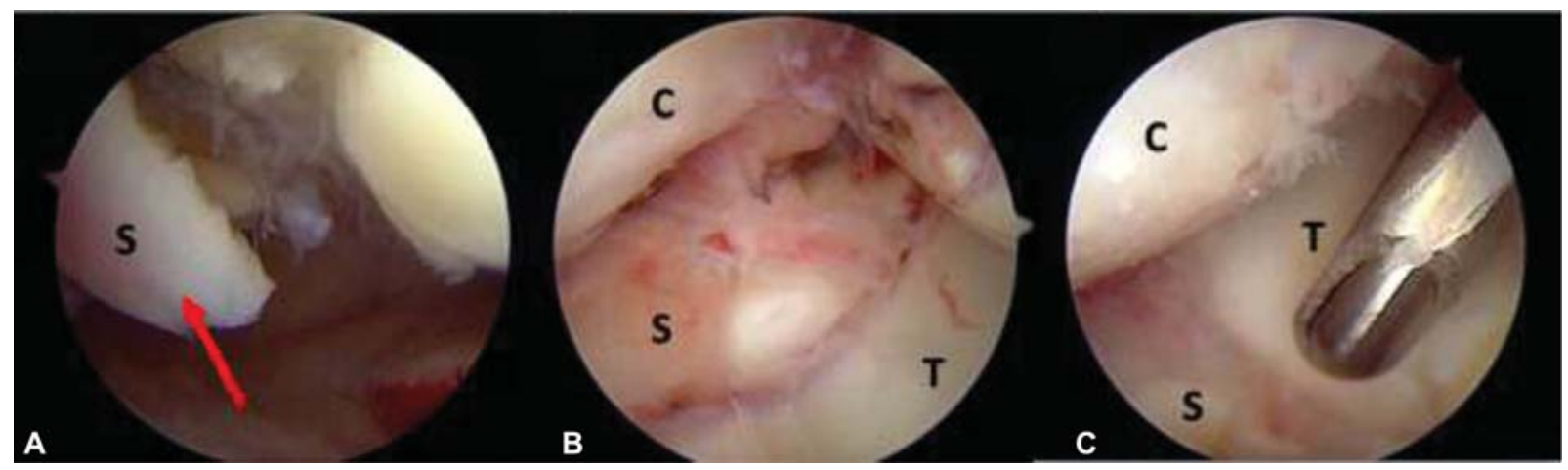

Figure 3 Diagnostic arthroscopic images during scaphocapitate arthrodesis. A) Visualization of the lunate fragmentation from the radiocarpal joint. The arrow shows a cartilage flap at the proximal lunate. B) Lunate joint cartilage damage visualized through the midcarpal joint. C) Capitate joint cartilage damage seen through the midcarpal joint. $S=$ Lunate, $C=$ Capitate, $T=$ Triquetrum. 
- Arthroscopic assistance for bone graft provision: The limb is repositioned for traction and an arthroscopy of the midcarpal joint is repeated. Through the MCR and MCR-A, the previously loaded morselized graft is introduced into an arthroscopic sleeve similar to the one used at the previously denuded scaphocapitate space (-Figures 7 and 8).

- Scaphocapitate screws fixation: The traction device is removed, and the limb is horizontally positioned with the wrist in pronation. Using the fluoroscope, the passage of the scaphocapitate guidewires is completed and both arthrodesis screws are implanted. As previously mentioned, we use a $3.0-\mathrm{mm}$ distal and a $2.4-\mathrm{mm}$ proximal headless screws. The correct positioning of screws and the graft is checked under fluoroscopy (-Figure 9).

\section{Postoperative Rehabilitation}

A removable wrist immobilizer is used for 2 weeks (until suture removal) and then a molded short arm cast is placed for 4 weeks. At 6 weeks, initial consolidation is assessed in a CT scan. If bone bridges are detected, the patient is referred to kinesiotherapy. Rehabilitation is performed until accepted functional ranges are recovered and there is no pain.

This technique has yielded good clinical-imaging outcomes in a series of six operated patients. All presented with complete consolidation, significant pain improvement and joint ranges preservation (-Figure 10).

We believe that scaphocapitate arthrodesis is an effective technique in reducing pain symptoms and allowing limb function in these patients. In contrast to the open technique,

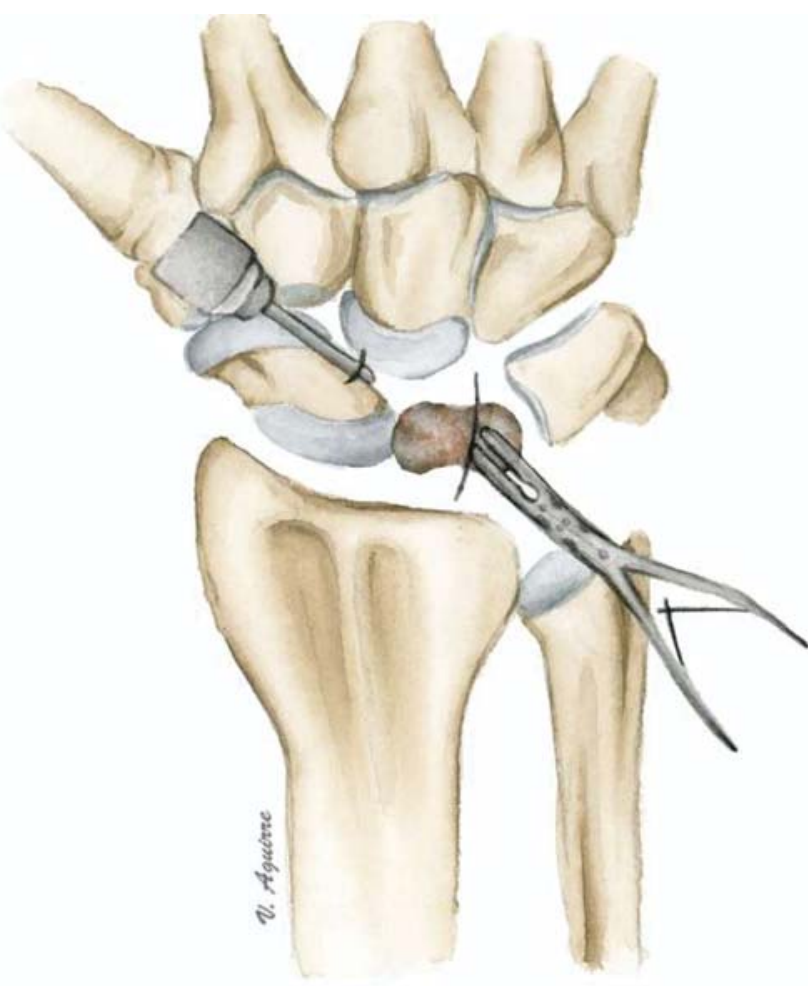

Figure 4 Scheme for lunate complete resection through a mini-open approach at the 3-4 portal. arthroscopic assistance has the advantage of being a minimally invasive procedure, reducing associated soft tissues damage, improving postoperative rehabilitation, and providing better cosmetic results. ${ }^{8}$

\section{Management in Terminal Stages}

Patients with complete lunate involvement and carpal collapse (Lichtman stage IV) are considered terminal cases. For them, surgical management consists of salvage surgeries. We propose arthrodesis or total wrist arthroplasty as the proper surgical options for these patients. The objective in these cases is clearly to reduce pain and regain limb function.

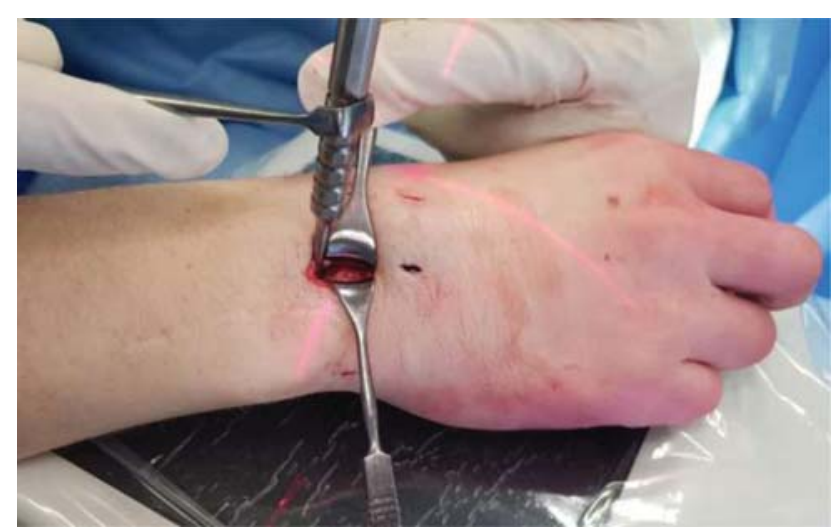

Figure 5 Mini-open approach for lunate extraction.

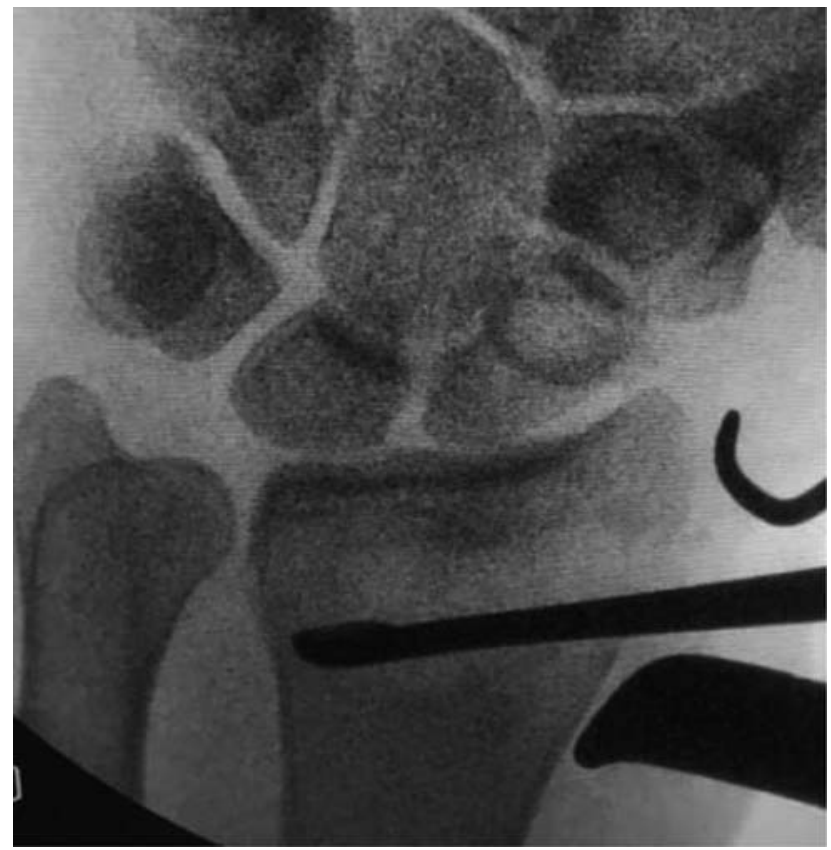

Figure 6 Intraoperative radiographic image of the distal radius graft and Illarramendi technique. 


\section{Conclusion}

Kienböck's disease represents a challenge for hand surgeons because it is a complex condition that generates wrist pain and loss of function in young, mainly working people who require timely treatment to achieve the best clinical outcomes. ${ }^{2,4,5}$ Advanced stages, with generalized carpal osteoarthritis, are often managed with salvage surgeries such as wrist arthroplasty or total wrist arthrodesis. ${ }^{4,7,11}$ For earlier stages, there is no consensus on the best treatment and the literature describes different surgical techniques to reduce the pain caused by synovitis and destruction while trying to preserve as much function as possible. ${ }^{7,11}$

In this context, partial carpal arthrodesis seems to be a proper option with good outcomes reported in the literature. ${ }^{2-8,11}$ Scaphocapitate arthrodesis reduces the load on the middle column by transferring it to the radial column, thereby reducing pain while preserving some degree of wrist mobility. ${ }^{7}$

Ho et al. ${ }^{8}$ describe different types of arthroscopic partial carpal fusions with good clinical outcomes and suggest this method as optimal for patients with limited carpal osteoarthritis who wish to preserve function. Although it is a demanding technique, it has a low rate of complications and high rates of union, with consolidation times comparable to those obtained with open techniques.

We propose a therapeutic scheme according to the Lichtman and Bain/Begg classification systems ( - Figure 11).

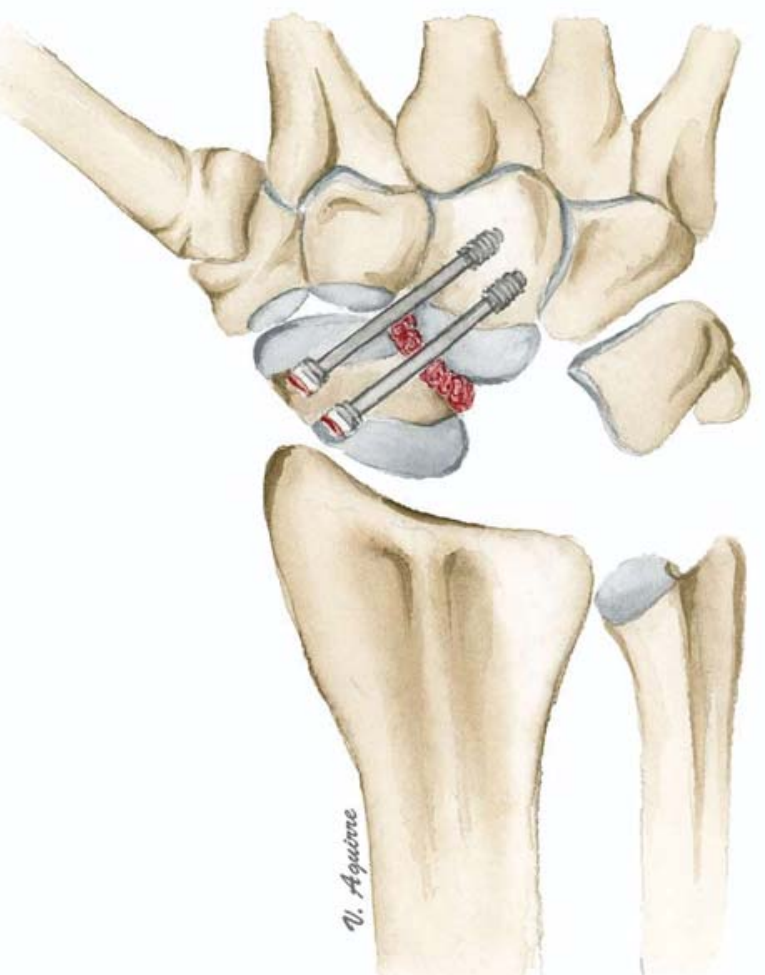

Figure 7 Scheme for scaphocapitate arthrodesis with two cannulated headless screws and a morselized graft.

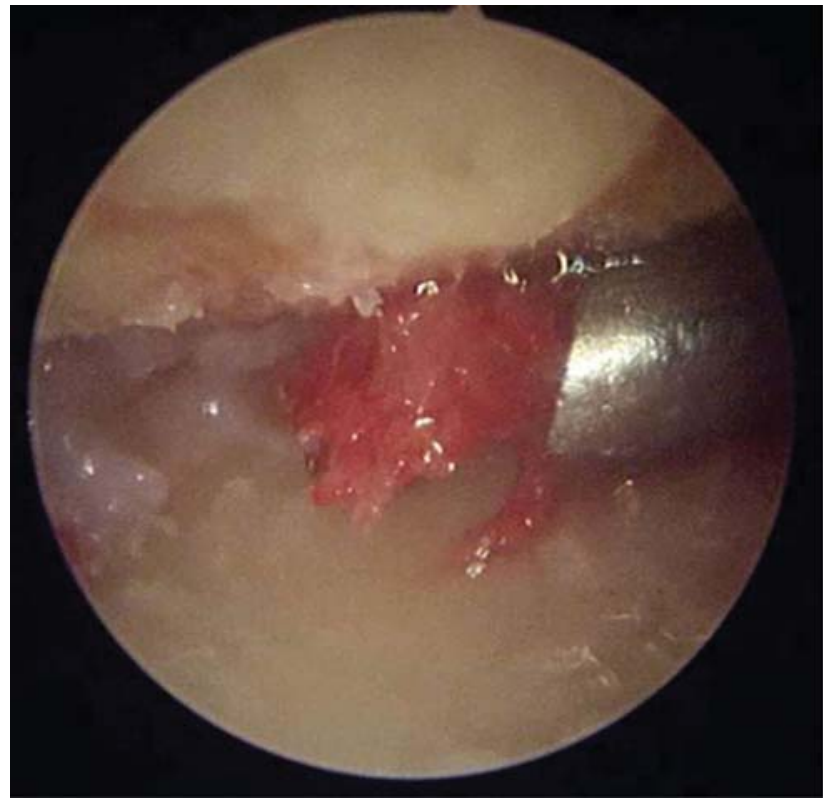

Figure 8 Arthroscopic image from the introduction of a morselized graft into the scaphocapitate space.

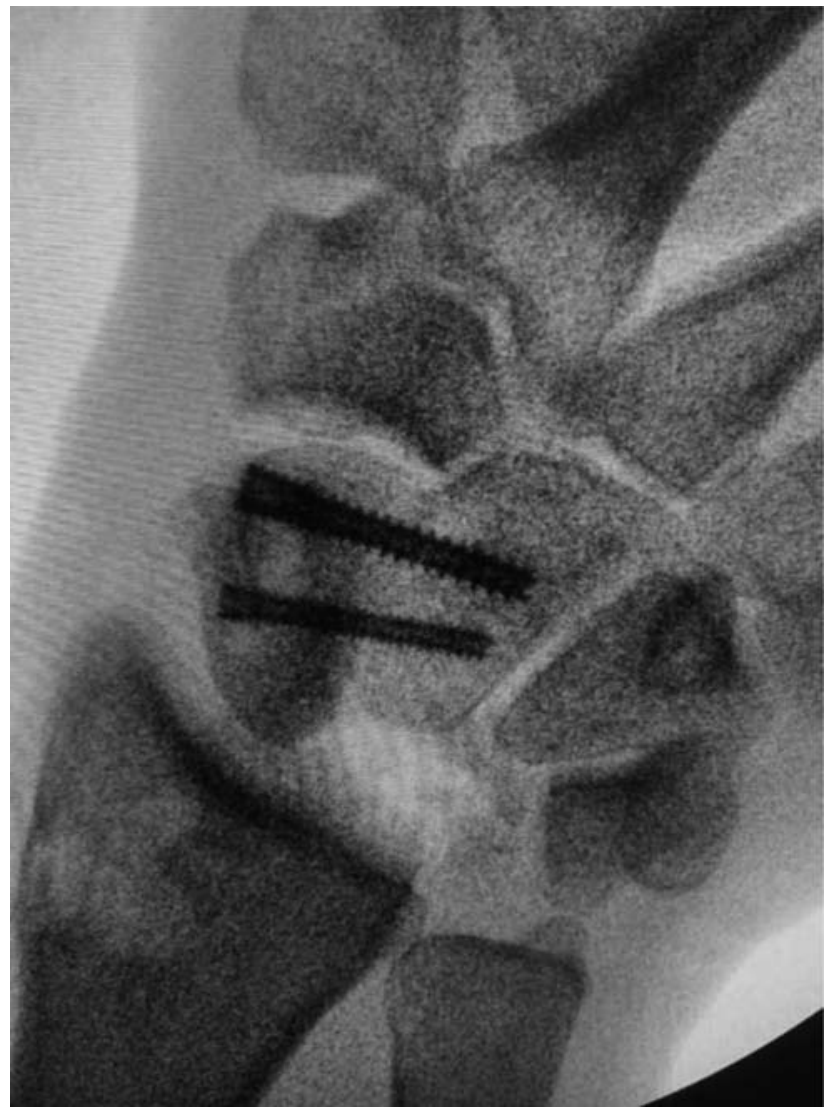

Figure 9 Fluoroscopic view of the scaphocapitate arthrodesis with screws. 

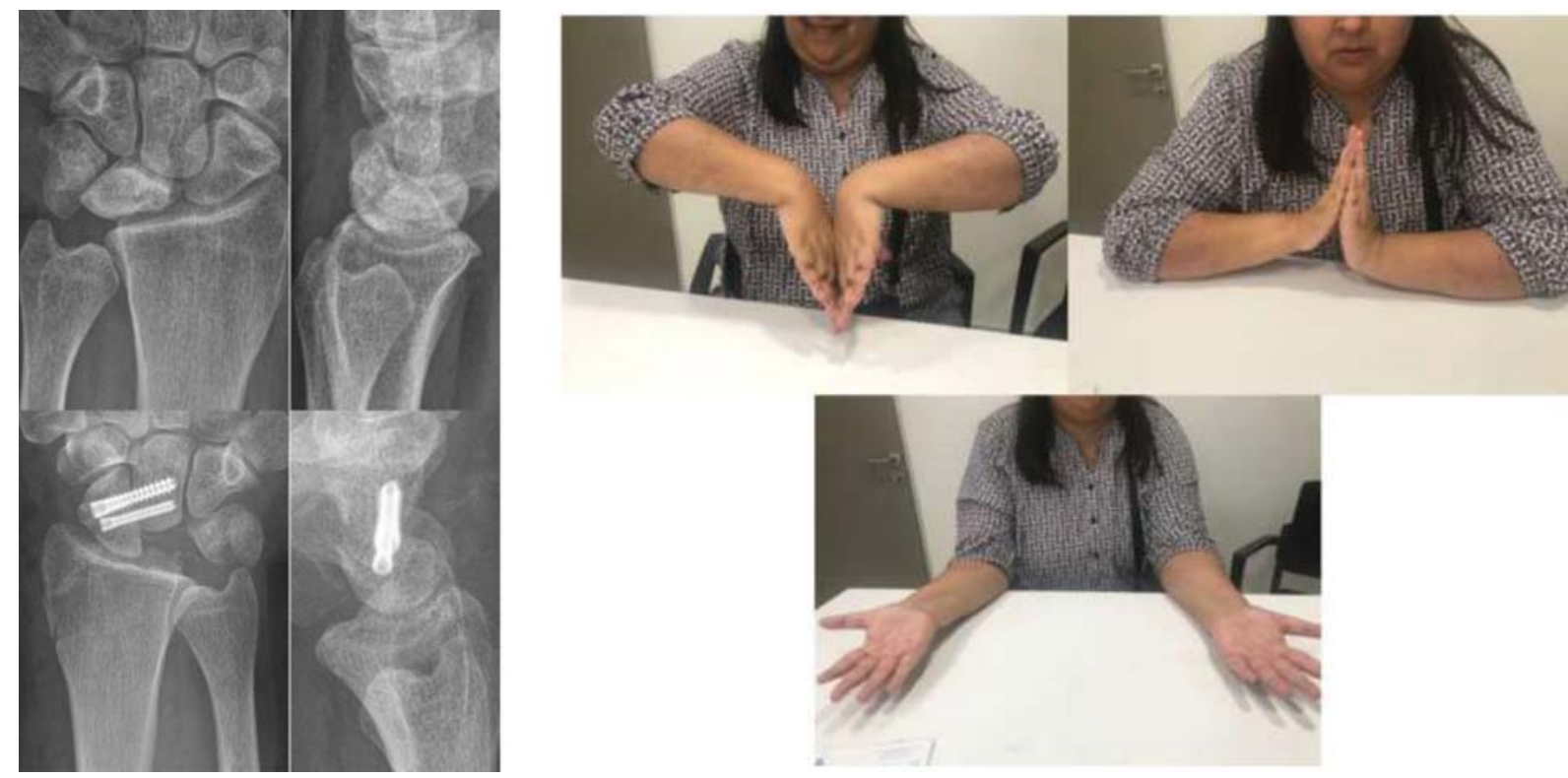

Figure 10 Follow-up of a patient submitted to a scaphocapitate arthrodesis with arthroscopic assistance 6 months ago. Joint ranges and the pre- and postoperative radiographs are shown.

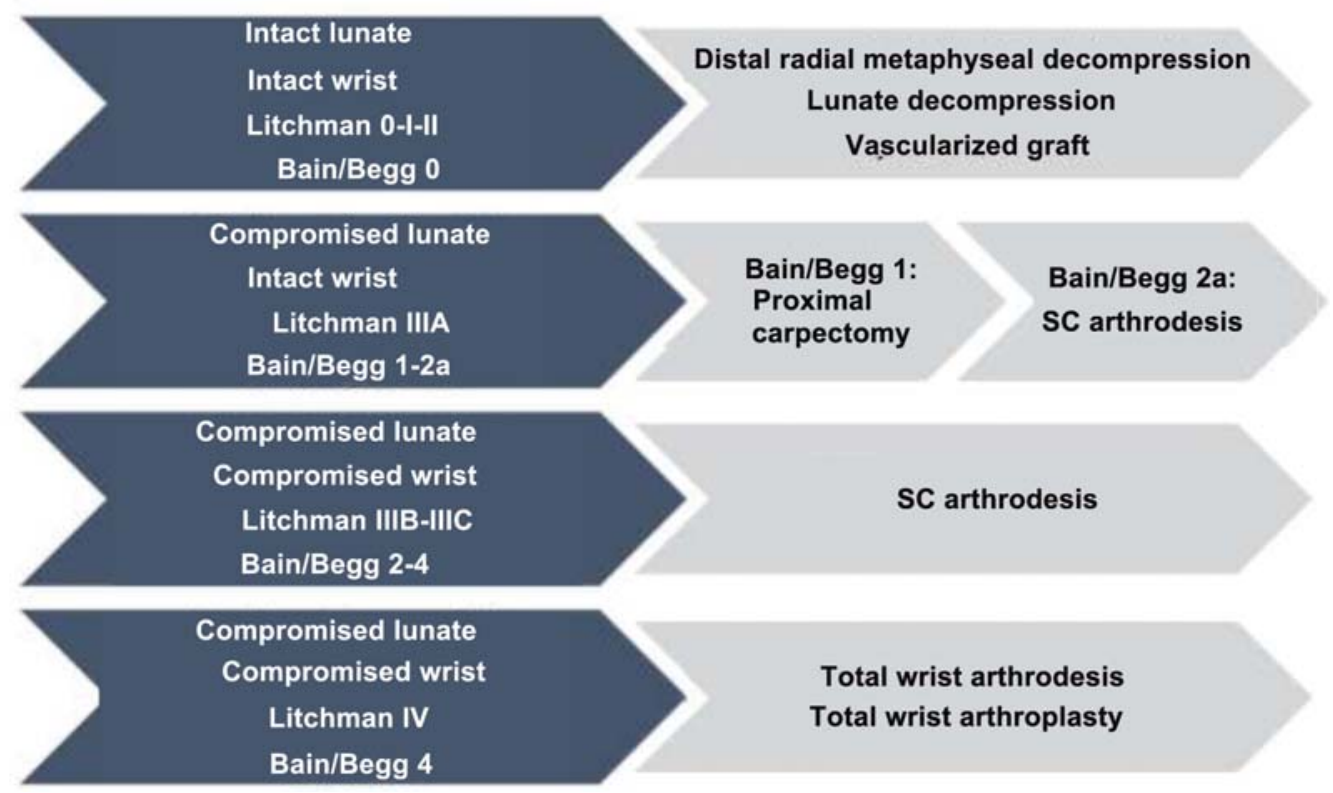

Figure 11 Surgical treatment scheme proposed by the authors according to lunate and wrist involvement. * SC = Scaphocapitate.

\section{Conflict of Interests}

The authors have no conflict of interests to declare.

\section{References}

1 Bain GI, MacLean SB, Yeo CJ, Perilli E, Lichtman DM. The Etiology and Pathogenesis of Kienböck Disease. J Wrist Surg 2016;5(04): 248-254

2 Leblebicioğlu G, Doral MN. Atay A öA, Tetik O, Whipple TL Open treatment ofstageIIIKienböck's disease with lunate revascularization compared with arthroscopic treatment without revascularization. Arthroscopy 2003;19(02):117-130
3 Lichtman DM, Pientka WF II, Bain GI. Kienböck Disease: A New Algorithm for the 21st Century. J Wrist Surg 2017;6(01):2-10

4 Fontaine C. Kienböck's disease. Chir Main 2015;34(01):4-17

5 Ertem K, Görmeli G, Karakaplan M, Aslantürk O, Karakoç Y. Arthroscopic limited intercarpal fusion without bone graft in patients with Kienböck's disease. Eklem Hastalik Cerrahisi 2016;27(03):132-137

6 Iorio ML, Kennedy CD, Huang JI. Limited intercarpal fusion as a salvage procedure for advanced Kienbock disease. Hand (N Y) 2015;10(03):472-476

7 Bain GI, MacLean SB, Tse WL, Ho PC, Lichtman DM. Kienböck Disease and Arthroscopy: Assessment, Classification, and Treatment. J Wrist Surg 2016;5(04):255-260 
8 Ho PC. Arthroscopic partial wrist fusion. Tech Hand Up Extrem Surg 2008;12(04):242-265

9 Geutjens GG. Kienböck's disease in an elderly patient. J Hand Surg Am 1995;20(01):42-43

10 Beckenbaugh RD, Shives TC, Dobyns JH, Linscheid RL. Kienböck's disease: the natural history of Kienböck's disease and consideration of lunate fractures. Clin Orthop Relat Res 1980;(149): 98-106

11 Bain GI, Begg M. Arthroscopic assessment and classification of Kienbock's disease. Tech Hand Up Extrem Surg 2006;10(01):8-13

12 Lichtman DM, Degnan GG. Staging and its use in the determination of treatment modalities for Kienböck's disease. Hand Clin 1993;9(03):409-416

13 Gelberman RH, Bauman TD, Menon J, Akeson WH. The vascularity of the lunate bone and Kienböck's disease. J Hand Surg Am 1980;5 (03):272-278

14 Stahl F. On lunatomalacia (Kienböck's disease): clinical and roentgenological study, especially on its pathogenesis and late results of immobilization treatment. Acta Chir Scand 1947;95 (Suppl 126):1-133

15 Tatebe M, Koh S, Hirata H. Long-Term Outcomes of Radial Osteotomy for the Treatment of Kienböck Disease. J Wrist Surg 2016;5 (02):92-97

16 Illarramendi AA, De Carli P. Radius decompression for treatment of kienböck disease. Tech Hand Up Extrem Surg 2003;7(03): 110-113

17 De Carli P, Zaidenberg EE, Alfie V, Donndorff A, Boretto JG, Gallucci GL. Radius Core Decompression for Kienböck Disease Stage IIIA: Outcomes at 13 Years Follow-Up. J Hand Surg Am 2017;42(09): 752.e1-752.e6

18 Moran SL, Cooney WP, Berger RA, Bishop AT, Shin AY. The use of the $4+5$ extensor compartmental vascularized bone graft for the treatment of Kienböck's disease. J Hand Surg Am 2005;30(01): 50-58

19 Sutro CJ. Treatment of nonunion of the carpal navicular bone. Surgery 1946;20:536-540 